Revue internationale P.M.E.

Économie et gestion de la petite et moyenne entreprise

\title{
Organisation et compétitivité des systèmes locaux de production en Espagne
}

\section{Maria-Teresa Costa Campi}

Volume 9, numéro 3-4, 1996

URI : https://id.erudit.org/iderudit/1008270ar

DOI : https://doi.org/10.7202/1008270ar

Aller au sommaire du numéro

Éditeur(s)

Presses de l’Université du Québec

ISSN

0776-5436 (imprimé)

1918-9699 (numérique)

Découvrir la revue

Citer cet article

Costa Campi, M.-T. (1996). Organisation et compétitivité des systèmes locaux de production en Espagne. Revue internationale P.M.E., 9(3-4), 122-148.

https://doi.org/10.7202/1008270ar
Résumé de l'article

Cet article contient les résultats obtenus d'une recherche appliquée sur l'organisation et la compétitivité des systèmes locaux de production (SLP) en Espagne. On analyse l'incidence des économies externes sur l'efficience et sur la capacité d'innovation des entreprises situées dans un territoire historiquement et géographiquement déterminé. On démontre que les économies externes expliquent le modèle d'organisation industriel adopté, tout en relevant des différences dans la capacité d'innovation et dans l'internationalisation des SPL selon la nature des relations entre les entreprises locales et le marché final. 


\title{
Organisation et compétitivité des systèmes locaux de production en Espagne ${ }^{1}$
}

Maria-Teresa COSTA CAMPI

Université de Barcelone

\section{MOTS CLÉS}

\section{Économies externes - Spécialisation - Décentralisation productive - Petites entreprises - Coûts de transaction Partenariat - Systèmes locaux de production - Flexibilité Internationalisation}

\begin{abstract}
L'AUTEUR
Maria-Teresa Costa Campi détient un doctorat en économique de l'Université de Barcelone et est professeure d'économie appliquée à la Faculté des sciences économiques de cette même université depuis 1987 . Ses plus récentes publications sont: 1) Cooperación industrial entre pequeñas y medianas empresas. Criterios de selección e instrumentos de fomento, (1992, Madrid, MICYT, en collaboration avec M. Callejón), 2) Cooperación entre empresas y sistemas productivos locales en España. Una approximación (1993, Madrid, IMPI) et 3) Ejercicios y prácticas de Economía Española (1994, Éditorial Civitas). Elle a publié un grand nombre d'articles dans des revues scientifiques comme Información Comercial Española; Économie et Société; Economistas et Revista Económica de Catalunya. Adresse: Université de Barcelone, Département d'économique, Faculté des sciences économiques, Av. Diagonal, 690, 08034, Barcelone.
\end{abstract}

1. Cet article repose sur une étude de la coopération entre les entreprises et les systèmes locaux de production. J'adresse mes remerciements au ministère de l'Industrie dont le soutien financier a permis la réalisation d'un Programme d'Excellence au sein du C.E.P.

Ma reconnaissance va également à l'équipe de recherche du C.E.P. pour son soutien, sans lequel cette étude appliquée n' aurait pu être complétée.

J'apprécie la revue et les suggestions faites par Pierre-André Julien pour cette étude ainsi que l'aide offerte par ma collaboratrice Carolina Hintzmann. 


\section{RÉSUMÉ}

Cet article contient les résultats obtenus d'une recherche appliquée sur l'organisation et la compétitivité des systèmes locaux de production (SLP) en Espagne. On analyse l'incidence des économies externes sur l'efficience et sur la capacité d'innovation des entreprises situées dans un territoire historiquement et géographiquement déterminé. On démontre que les économies externes expliquent le modèle d'organisation industriel adopté, tout en relevant des différences dans la capacité d'innovation et dans l'internationalisation des SPL selon la nature des relations entre les entreprises locales et le marché final.

\section{ABSTRACT}

This article shows the results of an applied research study about the organization and competitivity in local production systems (LPS) in Spain. It analyses the incidence of external economies on the efficiency and innovation ability of enterprises located in a both historically and geographically determined territory. It proves that external economies can explain the adopted model of industrial organization, although some differences may arise in innovation and internationalization ability of LPS depending on the nature of the existing relations between enterprises and the final market.

\section{RESUMEN}

En este articulo se recojen los resultados obtenidos en una investigación y competitividad de los sistemas productivos locales (SPL) en España. Se analiza la incidencia de las economias externas en la eficiencia y capacidad de innovación de las empresas localizadas en un territorio historica y geográficamente determinado. Se demuestra que las economias externas explican el modelo de organizatión industrial adoptado, si bien se observan diferencias en la capacidad innovadora y en la internacionalización de los SPL en función de la naturaleza de las relaciones entre las empresas locales y el mercado final. 


\section{Les conditions d'organisation de l'efficacité dans les systèmes de production locaux}

La décentralisation et l'extension de l'industrialisation à l'intérieur d'un territoire donné s'opèrent à la suite de l'apparition d'unités spécialisées de production de taille limitée et de l'externalisation des fonctions par les grandes entreprises ; ces fonctions sont alors intégrées aux systèmes locaux de production. Dans les deux cas, les relations entre les entreprises se situent au niveau de la recherche, de la production et de la commercialisation.

Dans les modèles décentralisés, les entreprises établissent des relations de coopération fondées sur la complémentarité et l'existence d'économies d'échelle autant au niveau des entreprises que de l'industrie. Ainsi, la densité des structures sociales de production et le développement du marché local déterminent l'efficacité des systèmes industriels décentralisés.

Afin d'expliquer l'efficacité organisationnelle des systèmes décentralisés, il est nécessaire d'avoir à l'esprit des facteurs tels que la capacité à générer et à développer les innovations, la coopération, l'abondance du capital humain sur les marchés locaux de main-d'œuvre, l'accès à l'information, la mobilité et la cohésion socio-industrielle. Cela signifie qu'on doit partir d'un ensemble de variables gravitant autour de l'entreprise, sans lesquelles ni l'entreprise ni le système entrepreneurial local ne serait en mesure d'acquérir une position concurrentielle sur le marché. La concrétion et la modernisation du concept d'économies externes nous permettent de faire une distinction entre les économies spécialisées découlant de la division du travail entre les entreprises dans un système local de production, les économies d'information et de communication équivalentes à la notion de coûts de transaction et les économies d'offre de main-d'œuvre provenant de la formation du potentiel de main-d'œuvre locale (Zeitin, 1992).

La notion d'économies externes, telle qu'elle a été introduite par Marshall, explique les bénéfices croissants liés à la décentralisation de la production. Ainsi, certains auteurs, tels Young (1928) et Stigler (1951), poursuivant la tradition marshallienne, ont associé les profits croissants aux économies engendrées par la spécialisation. Par ailleurs, ils expliquent essentiellement l'organisation industrielle soit par la désintégration des processus complexes de production en séries de processus simples ou, encore, par les liens économiques externes au processus de production, et plus particulièrement par l'expansion du marché. La décentralisation du processus de production favorise la coopération entre des entreprises qui peuvent être coordonnées à travers les mécanismes du marché (Dei Ottati, 1988). 
Fondée sur la complémentarité entre les entreprises, la coopération dont il est fait mention ci-dessus suppose l'existence et la création d'un groupe d'économies externes positives de production qui, dans certains cas, peuvent requérir que des transactions soient menées et des accords conclus. La formalisation de ces accords engendre, dans la plupart des cas, des coûts appelés «coûts de transaction».

L'incidence des variables institutionnelles sur l'organisation des processus de production a été étudiée par quelques auteurs comme Williamson et Stigler. L'interprétation technologique de Stigler trouve son contraste dans les travaux de Williamson (1975) sur les marchés et les hiérarchies. Williamson soutient que ce ne sont pas les considérations liées à la technologie, mais plutôt celles liées aux coûts de transaction qui expliquent les processus d'intégration verticale de la production. L'explication fondée sur les transactions postule que les économies d'échelle ne sont pas pertinentes. Aussi, le choix entre l'internalisation, d'une part, et l'externalisation, de l'autre, doit entièrement dépendre des différences dans les coûts de transaction (Williamson, 1985).

Pour Williamson, l'intégration verticale, ou alternativement la décentralisation de la production, sera déterminée non par des raisons d'ordre technologique (bien que celles-ci puissent avoir un effet secondaire), mais par les coûts de transaction. De là, on peut déduire que la technologie a des caractéristiques de séparabilité qui permettent de définir des domaines de production coordonnés dans le cadre d'organisations de remplacement.

Les approches respectives de Stigler et de Williamson représentent les apports théoriques les plus viables à l'explication des causes qui déterminent les systèmes d'organisation intégrés et décentralisés. Toutefois, on doit noter, en premier lieu, que la position transactionnaliste, qui est la plus répandue au sujet de la dichotomie entre le marché et la hiérarchie, est extrêmement simplificatrice et, en second lieu, que les approches de Stigler et de Willliamson ne sont pas nécessairement exclusives.

La formulation précédente a été révisée en partie par Williamson dans ses plus récents travaux (Williamson, 1985 ; Aoki, Gustavsson et Williamson, 1990). Dans ces études, Williamson renforce les thèses institutionnalistes au sujet de l'incidence de la confiance sur la diffusion de l'information. Cela confère à la coopération un rôle en tant que lieu central de liaisons dans les organisations formelles et informelles.

La nouvelle approche élaborée par Williamson indique que les entreprises peuvent trouver des incitatifs à coopérer. Si la coopération est, comme le soutient Axelrod (1984), basée sur le principe de réciprocité, alors les entreprises éviteront d'adopter un comportement opportuniste et bénéficieront des incitations à réaliser des transactions. En fait, Williamson et Axelrod raisonnent 
tous deux selon le «dilemme du prisonnier» en supposant que les facteurs environnementaux et humains permettent des résultats optimaux qui devraient être atteints par le biais de la collaboration plutôt que par la défection mutuelle.

En relation avec cette seconde réflexion que nous avons mise de l'avant, nous devons souligner que l'articulation entre les observations de Stigler et de Williamson peut être traitée dans le cadre d'un modèle dynamique de désintégration verticale. Les efforts théoriques réalisés dans cette voie (Bellandi, 1988) nous permettent d'avancer vers la définition du concept de décomposition technologique, qui est aussi présent chez Stigler et Williamson, et d'expliquer d'une manière convaincante les relations entre les économies d'échelle et la division du travail dans les structures industrielles en changement. Dans ce contexte, nous pouvons également conclure que les coûts de transaction seront influencés par l'existence d'économies d'échelle.

Aussi, la coopération industrielle bénéficiera non seulement de coûts de transaction peu élevés tout en mobilisant les externalités, mais permettra également de réaliser des économies d'échelle à travers le complexe industriel. Nous pouvons alors en déduire que l'efficacité organisationnelle des systèmes industriels décentralisés est fonction de la densité de la coopération entre les entreprises.

À cette étape-ci, il est facile de conclure que la collaboration entre les entreprises conduit à l'expansion du marché au même titre que l'accroissement et l'amélioration de la qualité des économies externes de production sont une condition nécessaire au maintien de la coopération. Considérer les systèmes de production décentralisés comme étant une nouvelle forme d'organisation industrielle pourrait permettre l'articulation de deux conceptions de l'entreprise, soit comme une fonction de production et comme un résultat des facteurs environnementaux et humains qui conditionnent les coûts de transaction, c'est-à-dire entre les approches technologiques et institutionnelles. Ainsi, la décentralisation de la production conduit le système vers un processus de spécialisation et de complémentarité créant une structure dense de relations interentreprises à la fois formelles et informelles. Toutefois, ces relations ne peuvent être expliquées que par la complémentarité et la flexibilité de la production à l'intérieur d'agglomérations d'économies d'échelle. L'existence de facteurs de nature institutionnelle qui permettent la formation de réseaux de coopération, constituant un nouveau niveau institutionnel, est aussi une condition nécessaire à l'efficience des systèmes décentralisés. Par conséquent, on peut soutenir qu'à l'intérieur d'un certain cadre de débat, les analyses technologiques et institutionnelles ne sont pas nécessairement opposées. Les avantages sociaux de la coopération sont réalisés à travers un processus de distribution du système de coopération lui-même. Plus le réseau des accords établis est étendu, plus grand est l'effet de dispersion des innovations réalisées et plus étendus sont les avantages pour la collectivité. 
Ici, nous devons considérer les implications de la division des fonctions dans l'industrie comme conséquences, d'une part, de l'accroissement de la spécialisation et des usines spécialisées individuelles en une seule fonction et, d'autre part, de l'existence et de la taille des nouveaux niveaux institutionnels.

\section{L'élément territorial comme facteur d'intégration des économies externes de production}

La cohésion sociale est un stimulant potentiellement puissant qui engendre un ensemble d'externalités positives pour les entreprises. Dans ce sens, le concept marshallien des économies externes constitue une catégorie théorique explicative du fonctionnement des systèmes décentralisés de production dans un contexte territorial déterminé, celui d'un district industriel. Ainsi, la dimension des nouveaux phénomènes d'industrialisation spécialisée et localisée en rapport avec le dynamisme entrepreneurial a nécessité le recours à des instruments analytiques non hiérarchiques qui intègrent les variables industrielles, sociales et territoriales. Ce n'est pas sans raison que l'économie, telle que Hicks l'a observée, est une discipline concernée par un monde changeant. Les travaux de Becattini (1975, 1979) ont donné lieu à des analyses théoriques et empiriques s'inscrivant dans la tradition marshallienne et soulignant que les approches technologiques et sectorielles ne conviennent pas à l'analyse des nouveaux phénomènes industriels. Comme nous l'avons déjà mentionné, l'étude de l'organisation industrielle des districts industriels (Marshall, 1980) nous permet de situer les précédents problèmes d'efficience et de compétitivité à l'intérieur d'une région territoriale déterminée.

Dans ce sens, les entreprises localisées dans une région donnée tirent des avantages mutuels de leur proximité. La localisation concentrée est une méthode non exclusive pour accroître la taille et la spécialisation dans une industrie. Les variables de nature industrielle, sociale et culturelle déterminent la formation de régions de spécialisation industrielle. À l'origine, ces concentrations industrielles se sont développées grâce à des groupes technologiques de transactions convexes et à l'inexistence de coûts externes. Cela se produit dans le temps en raison des avantages naturels, et toujours avec des retombées sociales et culturelles qui stimulent les économies externes et la propension au risque. Dans tous les cas, on doit noter que les avantages concurrentiels résultent des avantages dynamiques associés à l'activité et au changement.

L'expérience montre que la localisation d'entreprises spécialisées dans un territoire déclenche un ensemble de processus comme, par exemple, la croissance d'industries connexes et la formation d'infrastructures de base de recherche et de distribution, qui donnent à l'économie d'un territoire un niveau élevé de compétitivité et d'efficience. En même temps, la cohésion sociale facilite le 
développement de relations institutionnelles et la maximisation des effets de distribution des programmes de promotion de l'industrie et du développement social.

Les systèmes décentralisés et localisés ont également été étudiés à partir de la perspective de la stratégie concurrentielle. Porter (1990), à la suite de Marshall, considère la concentration géographique des systèmes de petites et moyennes entreprises (PME) comme un des modèles qui favorisent la compétition internationale. L'auteur démontre que la cohésion socio-industrielle et la flexibilité de production des régions de spécialisation industrielle facilitent le renforcement des avantages concurrentiels, définis en relation avec le caractère organique du système. Dans la même veine, $\operatorname{Krugman}(1991,1992)$ soutient que les entreprises locales spécialisées autour d'une activité suscitent des interactions complexes ainsi que le développement d'une culture sociale et de production, assurant alors un positionnement concurrentiel solide.

Nous devons induire de ce qui a été avancé que les systèmes de production locaux de PME spécialisées ont acquis ou sont en voie d'acquérir des avantages concurrentiels sur les marchés internationaux (Costa, 1991). Les bénéfices sociaux et économiques qui viennent avec ce modèle d'organisation industrielle sont soutenus par des perspectives héoriques et empiriques différentes. Par conséquent, ces économies qui démontrent les phénomènes de décentralisation locale, que ce soit pour des raisons théoriques ou parce qu'elles sont encore liées à des changements organisationnels plus récents, devraient en priorité considérer le renforcement des externalités locales positives.

\section{La taille et l'adaptabilité de la production comme un avantage concurrentiel}

Le processus de production implique la transformation d'un ensemble d'intrants et d'extrants. Ce processus peut être réalisé intégralement à l'intérieur de l'entreprise ou encore avec la participation de différentes entreprises qui entretiennent entre elles des relations d'affaires ou des accords de coopération. Le rôle de l'atmosphère industrielle locale et l'avantage concurrentiel de chaque fonction de production sont d'autant plus importants que la décentralisation de la production est poussée. De la même façon, le positionnement concurrentiel de chaque entreprise spécialisée dépendra non seulement des ressources spécifiques qu'elle possède, mais aussi, dans une certaine mesure, de ses ressources génériques et des ressources du milieu.

La décentralisation du processus de production entre les PME conditionne le modèle d'organisation à mettre en place, les stratégies à déployer et la compétitivité de chacune des firmes participantes. Au fur et à mesure que les entreprises établissent des critères d'organisation régulant le milieu externe, 
ce dernier ne provoque plus des variations inattendues. La sensibilité des systèmes décentralisés face aux variations du contexte explique l'adoption de systèmes permettant la coordination des fonctions de production désagrégées.

L'interrelation des ressources spécifiques et génériques entre les entreprises et le milieu donne lieu à une situation où le système, ou la fonction de production, devient l'assise des systèmes locaux industriels.

La fonction de production devient une variable concurrentielle fondamentale pour l'entreprise et pour l'ensemble du groupe formant le système local de production. Son importance vient essentiellement du fait que la fonction de production reflète et intègre le progrès et le changement technique qui sont à l'origine de la croissance et du développement des systèmes locaux de production (Fernandez et Fernandez, 1988).

Il est important de mentionner le dilemme entre l'efficience et l'efficacité. L'efficience a été traditionnellement la variable clé de la fonction de production. Pendant longtemps, l'optimisation de la production, conçue pour l'essentiel comme résultant de l'efficience du système de production, a été considérée comme le principal objectif de l'entreprise. Cette attitude a changé. Certains auteurs, tels Skinner (1978) et Gozzi (1987), ont insisté sur le fait que la fonction de production ne doit pas être subordonnée qu'à l'atteinte de l'efficience, mais aussi à celle de l'efficacité ; cela devant être compris comme la capacité du système de production à répondre de façon satisfaisante aux changements de la demande. Peu d'auteurs ont tenté de trouver une solution de synthèse au dilemme que posent l'efficience et l'efficacité. On a associé l'efficacité au concept de délai de mise en production (lead-time), c'est-à-dire le temps qui s'écoule entre l'apparition d'une nouvelle demande et le moment où le système de production est en mesure de la satisfaire (Mariotti, 1982).

L'organisation flexible de la production peut engendrer des coûts et rendre inefficientes les fonctions qui sont opérées. Néanmoins, cette inefficience peut aussi être accompagnée de retombées positives croissantes à condition que les mécanismes concurrentiels du marché soient régulés par rapport à la qualité et non au prix (Fernandez et Fernandez, 1988). Autrement dit, les systèmes de productions flexibles et discontinues sont concurrentiels pourvu que les consommateurs soient disposés à payer un prix plus élevé pour obtenir des produits personnalisés de qualité. Il est donc nécessaire d'apprendre comment gérer un système flexible de production avec tout ce que cela implique, comme par exemple la diversité et la discontinuité de la production.

Cela dit, nous pouvons avancer que l'on retrouve les entreprises dans les systèmes de production locaux où les fonctions sont désagrégées, l'élasticité du revenu est élevée, le cycle de vie des produits est court, les marchés sont segmentés et turbulents et où une cohésion avec le milieu existe. 


\section{La réponse de la production aux changements de la recherche et le développement dans les systèmes locaux}

Dans la majorité des systèmes complexes de production locaux (Brusco, 1992), les facteurs de flexibilité coïncident, en relation avec le contexte local (flexibilité locale), avec les facteurs de nature technologique et avec ceux liés à la capacité et à l'ajustement saisonnier. Cette organisation flexible du processus de production nous permet de relever les caractéristiques suivantes (Katz, 1984 ; Fernandez et Fernandez, 1998):

1) usage multiple des biens détenus en commun et développement des marchés d'équipements de seconde main ;

2) capacité à répondre aux demandes individuelles et évolutives, adaptabilité ;

3) qualification élevée de la main-d'œuvre ;

4) coûts variables de production élevés ;

5) absence d'économies d'échelle ;

6) contrôle collectif du processus de production ;

7) diffusion de l'information par le biais des réseaux créés par les relations de production décentralisées.

L'adaptabilité des fonctions flexibles de production confère à ces systèmes une forte capacité d'innovation, en plus du processus d'innovation qui découle de leur propre modèle organisationnel. Dans les systèmes flexibles de production, le développement de nouveaux produits s'effectue en fonction de la production et de la sélection d'idées provenant de sources d'information externes, en particulier les clients, les consommateurs, les fournisseurs et les marchés où les innovations sont diffusées (foires commerciales et congrès).

Les différentes phases du processus de développement des nouveaux produits tendent à être externalisées. Cette décentralisation est induite à la fois par le développement du marché local et par le seuil élevé du coût d'efficacité de ces activités. La rapidité du processus de changement technologique a engendré de fortes réductions du temps de maturation des projets d'innovation. Enfin, il faut souligner que les caractéristiques organisationnelles des systèmes flexibles de production font que les innovations consistent généralement en l'introduction de nouvelles lignes de produits sur des marchés déjà existants.

Les recherches effectuées sur les changements dans la fonction de production (Lorenzoni, 1980, Fernandez et Fernandez, 1998 ; Gozzi, 1987 ; Tani, 1987) montrent que l'externalisation revêt un sens plus large que celui qui se dégage 
de la simple notion de décentralisation. L'accélération des processus d'innovation, l'intégration dans la production de la technologie informatique, la complexité accrue du marché, les changements continus de la demande et l'émergence de divers mécanismes de compétition à partir du prix ont mené à la formation d'accords de coopération visant à rechercher et à créer des synergies externes. En effet, la concentration des économies externes engendre des synergies intenses au sein des groupes d'entreprises qui entretiennent des accords de coopération. De cette manière, l'entreprise ou encore le groupe d'entreprises le plus fort dans le système local peut faire face aux défis du marché, sa compétitivité résultant des économies externes de synergie.

\section{Les résultats de l'étude empirique}

En utilisant le modèle théorique décrit antérieurement, une étude empirique a été réalisée sur l'organisation du processus de production, la capacité d'innovation et leur mode de développement, l'ajustement des marchés locaux de main-d'œuvre et les économies externes locales de production. La recherche a été menée dans 23 régions couvrant 234 entreprises industrielles qui opèrent dans le groupe d'activités dominantes de chaque région. Le critère de sélection de l'échantillon fut non aléatoire. L'insuffisance des données statistiques individuelles et courantes sur les entreprises industrielles a empêché une sélection aléatoire; celle-ci fut plutôt basée sur des informations privilégiées fournies par des experts provenant de chaque région ${ }^{2}$.

\subsection{L'organisation des processus de production dans les systèmes locaux}

L'analyse des données issues des informations obtenues de l'échantillon étudié nous a permis d'avoir une meilleure compréhension des caractéristiques de l'orga-

2. Les 23 régions ont été choisies en fonction d'études antérieures, à caractère historique et industriel, qui ont montré l'existence de concentrations de PME spécialisées dans des activités complémentaires liées au territoire. Les 23 cas incluent des activités différentes. Ces régions ne constituent pas toutes des districts industriels dans le sens de Marshall-Becattini, mais elles présentent néanmoins plusieurs caractéristiques, étudiées dans cet article, qui permettent de les considérer comme des systèmes locaux de production ou des systèmes de zones conformément à la perspective de Garofoli. L'approche méthodologique, basée sur des critères non aléatoires, est justifiée par le fait que l'Espagne manque de répertoire industriel qui aurait permis de déterminer tous les systèmes de production locaux au moyen d'une analyse factorielle. Quelques études réalisées par plusieurs chercheurs ont permis d'identifier 143 systèmes locaux. Toutefois, ces études ne recourent pas à la même méthodologie. La recherche présentée dans cet article est la première à avoir développé une analyse du comportement concurrentiel d'un large groupe de systèmes de production locaux. 
nisation de la production de ces firmes, considérant l'incidence des économies externes locales sur le modèle organisationnel et les relations de coopération entre les entreprises.

Les résultats des analyses empiriques ne permettent cependant pas de tirer des conclusions sur les caractéristiques des entreprises considérées sur une base individuelle et en relation avec leur appartenance à une agglomération industrielle dans une région locale. Dans cette perspective, l'entreprise est une unité de production efficiente dans la mesure où elle n'a pas besoin d'internaliser techniquement les fonctions de production nécessaires, en ayant autour d'elle les marchés complémentaires à ses activités et dont l'accès n'implique pas des coûts de transaction importants.

Les activités que ces entreprises réalisent sont déterminées par la structure industrielle de chaque région. Cette structure et l'organisation du processus de production ont été considérées ensemble dans l'échantillon non aléatoire.

L'échantillon regroupe les activités de production complémentaires dans chaque système de production local. La spécialisation des firmes implique que le processus complet de transformation est assuré grâce à la coordination, à travers le marché et les accords de coopération, de chaque phase de fabrication assumée par chacune des entreprises. Il est nécessaire d'insister sur le fait que la compétitivité et l'efficacité de la production sont réalisées au niveau de l'ensemble du système de production local et non à travers chaque entreprise, bien que chaque entreprise soutienne nécessairement les niveaux d'efficacité que requiert le fonctionnement équilibré et harmonieux de l'ensemble du groupe d'entreprises locales.

Aussi, l'analyse désagrégée par produit et par entreprise reflète-t-elle un degré élevé de spécialisation et de complémentarité entre les entreprises de chaque système de production. Les activités industrielles sont réalisées au sein du système de production comme ci-après :

- Machine-outil (Bajo Deba)

- Meubles (Urola)

- Logiciel (Sabadell)

- Composants pour l'aéronautique (Région de Sur (Madrid), Dos Hermanas Corridor)

- Confection de vêtements (Vallecas, Orense)

- Textile (Béjar, Alcoy)

- Transformation de la viande (Guijuelo)

- Jouets (Ibi)

- Fabrication métallique (Iscar) 
- Chaussures (Inca, Menorca, Elda, Fuensalida)

- Tenues de bijouterie (Menorca)

- Ingénierie électrique et composantes de moteurs de voiture (Sud de Madrid, Manresa)

- Conserves de légumes (Murcia)

- Accessoires en cuir (Ubrique)

- Travaux de marbre (Macael)

- Chaussettes (Mataro)

- Céramique (Castellon)

- Composantes pour bateaux (Vigo).

En tout, 19 activités industrielles, 544 produits et leurs marchés spécialisés ont été considérés dans cette étude.

La majorité des entreprises étudiées destinent leurs activités au marché. Elles manifestent un comportement différent de celui des systèmes voués à la fabrication de composantes destinées aux marchés oligopolitiques et de ceux dont les activités sont à faible valeur ajoutée au niveau de la phase de production comparativement à la distribution. Dans ces derniers cas, les relations de sous-traitance sont organisées selon un modèle hiérarchique isolé de la concurrence ou des clients des autres marchés et empêchent les entreprises soustraitantes d'avoir un accès direct au marché. C'est le cas des fabricants dans l'industrie de l'aéronautique et des composantes pour moteurs de voiture qui ont peu de relations directes avec le marché et qui manifestent une plus grande dépendance à l'égard d'un petit nombre de clients uniques. Les systèmes de production de Dos Hermanas Corridor et, à un moindre degré ceux du Sud de Madrid et de Vigo font exception à cela. Dans le second groupe, bien que dans une faible proportion, on retrouve les entreprises de fabrication de vêtements de Vallecas. Comme nous le verrons plus loin, la relation inverse est observée entre la proximité au marché, soit la dépendance à l'égard d'un client, et la capacité d'innovation de l'industrie textile locale.

Les marchés locaux visés par ces entreprises manifestent un degré élevé de variation de la demande. Ainsi, 87,2\% d'entre elles ont une demande fluctuante à l'égard de leurs produits, et $61,9 \%$ d'entre elles connaissent des difficultés techniques au niveau des processus de production. Les cas les plus extrêmes apparaissent dans les systèmes de production locaux dédiés à des activités liées à la mode ou à la consommation saisonnière, comme c'est le cas pour la fabrication de vêtements (Vallecas), le textile (essentiellement à Béjar), les jouets (Ibi), les tenues de bijouterie (Menorca), les chaussettes (Mataro) et la chaussure (essentiellement à Elda et Fuensalida). Le système de production local ayant le marché le plus stable et, par conséquent, le moins utilisé 
pour l'adoption de l'organisation et les changements de produits, est celui de Dos Hermanas Corridor.

Finalement, nous observons que les systèmes locaux à processus complexes de production, ceux qui fabriquent des produits très spécifiques et ceux dont l'organisation de la production est intégrée verticalement (conséquence de la faiblesse des économies externes locales) souffrent de difficultés techniques lorsqu'ils doivent répondre aux variations de la demande pour leurs produits. Les entreprises qui démontrent une plus grande sensibilité parmi les trois mentionnées sont, premièrement, celles des machines-outils à Bajo Deba et, deuxièmement, celles des jouets à Ibi. Ces derniers produits ont un cycle de vie très court en raison de la complexité de leurs procédés de production. Le troisième groupe d'entreprises est celui de la chaussure à Fuensalida, dont les difficultés techniques devraient être justifiées en raison (tel que nous l'avions déjà noté) des ressources industrielles locales encore limitées - faiblesse des économies externes - les empêchant de traiter avec les processus de décentralisation et la flexibilité.

Les résultats obtenus montrent que 47,5\% des entreprises choisissent de conclure des accords de coopération pour faire face aux variations de la demande de leurs produits.

La turbulence du marché et les conditions industrielles locales contribuent à la décentralisation d'une partie des processus de production. C'est pourquoi $74,3 \%$ des entreprises externalisent certaines fonctions de production que requiert le produit final. Ce processus de décentralisation favorise l'établissement de relations interentreprises sur des modes différents : 66,4\% des entreprises qui externalisent certaines fonctions de production le font à travers la sous-traitance, et de celles-ci, 12,7\% ont recours à d'autres formes de coopération.

La sous-traitance est la forme de relation interentreprises la plus fréquente dans le domaine de la production et chez celles dont les procédés de production sont à cycle long et nécessitent un grand nombre de composantes. Par conséquent, une relation claire ressort entre la densité et la spécialisation des structures industrielles locales et la sous-traitance. Les systèmes de production locaux où la sous-traitance occupe une plus grande place sont Bajo Deba, Urola, Sud de Madrid, Mansera et Vigo. Par contre, Sabadell, en raison des caractéristiques du marché et de la fabrication des logiciels, Vellecas, Menorca et Elda optent pour d'autres accords d'achat pour externaliser les fonctions de production complémentaires à leur activité principale.

L'activité organisationnelle adoptée par les entreprises et le contexte local peuvent expliquer la capacité élevée que démontrent les entreprises en matière d'innovation des produits et des procédés. En effet, 79,4\% d'entre elles 
affirment qu'au cours des trois dernières années elles ont entrepris des innovations de produits, alors que $71,4 \%$ ont réalisé des innovations de procédés. Ces entreprises financent ces innovations à même leurs propres ressources (autofinancement à 47,5\%).

Le but des innovations de produit, comme l'ont indiqué les entreprises, est d'améliorer la compétitivité et d'éviter de perdre la position occupée sur le marché compte tenu de celle des autres entreprises de la région. Nous pouvons donc conclure que la concurrence entre les entreprises sur les marchés locaux suscite la mise sur pied de projets innovateurs. Il est possible de relever l'effet du contexte dans l'origine géographique de l'information sur les innovations de produit, étant donné que $30,3 \%$ de l'ensemble des informations reçues (1 180) par ces entreprises proviennent de la même municipalité.

Les innovations de procédés, pour leur part, visent à améliorer la flexibilité et la spécialisation de l'entreprise. Ces améliorations répondent à la nécessité d'accroître la qualité, la capacité de production et la productivité.

La décentralisation des processus de production et la flexibilité organisationnelle favorisent en retour la multiplication des relations de coopération, au-delà de la simple sous-traitance. En effet, $50 \%$ des entreprises dans les systèmes locaux de production sont impliquées dans quelques types de coopération (tableau 1). Ces résultats révèlent une forte propension à coopérer. Dans ce sens, on peut penser que les coûts de transaction liés à la négociation ou à la gestion d'une collaboration sont faibles au niveau de la production, avec comme résultat que les entreprises réalisent une partie de leurs fonctions de production dans les régions environnantes et, au même titre, les fonctions de recherche et de service bien qu'à un moindre degré. L'existence d'accords informels renforce l'évidence des considérations avancées lors de l'analyse théorique et qui se rapportaient à l'incidence des économies externes sur la formation de flux d'intrants intangibles, bonifiant ainsi l'efficience organisationnelle des entreprises locales.

TABlEAU 1

Formes de coopération conclues par les entreprises

\begin{tabular}{lccc}
\hline Type de coopération & $\begin{array}{c}\text { Pourcentage } \\
\text { d'entreprise }\end{array}$ & $\begin{array}{c}\text { Accord } \\
\text { formel }\end{array}$ & $\begin{array}{c}\text { Accord } \\
\text { informel }\end{array}$ \\
\hline Établissement d'accords & $50 \%$ & $57,1 \%$ & $49,2 \%$ \\
Absence d'accord & $50 \%$ & & \\
Total des cas \% aux accords faits & & 324 & \\
Total des cas \% au type d'accord & & 182 & \\
\hline
\end{tabular}

Source : Costa, M.T. et al. (1993), Cooperación entre empresas y sistemas productivos locales en España. Una aproximación, Madrid, MINER. 
Globalement, les accords de coopération considérés sont concentrés au niveau de la production et, dans une certaine mesure au niveau de la distribution et de la R-D ${ }^{3}$. La collaboration entre les entreprises au niveau des services et du marketing est clairement moins élevée que celles dans d'autres sphères. Cependant, nous ne pouvons pas en inférer que les entreprises internalisent toujours ces fonctions, mais qu'elles ont en général moins de poids par rapport à l'ensemble des activités réalisées tant au sein de la firme prise individuellement qu' au sein du système de production local. Comme nous le soulignerons plus loin, la faiblesse des fournisseurs de services montre clairement la nécessité de promouvoir cette activité qui est décisive pour assurer la compétitivité du système de production.

Des typologies d'accords peuvent être relevées selon les domaines dans lesquels elles sont conclues. Les données recueillies révèlent une forte tendance à l'établissement d'accords formels dans le cas des activités de R-D, contrairement au poids relatif et absolu des collaborations informelles dans le domaine de la production. De même, l'interprétation des données obtenues révèle un comportement différent de la formule de celui adopté dans l'accord de coopération, suivant le domaine d'activité dans lequel il a été conclu. En général, la formule la plus fréquemment utilisée consiste en des accords temporaires entre les entreprises.

Les relations de coopération entre les entreprises dans les systèmes de production locaux s'établissent dans le contexte local entre des PME dans $91,9 \%$ des cas étudiés. Plus les PME sont spécialisées, en particulier dans la production, plus elles recourent au marché local.

Contrairement aux grandes entreprises, les unités de plus petite dimension ont moins tendance à coopérer à l'extérieur de leur milieu immédiat. Ainsi, la coopération internationale qui ne concerne que $7,8 \%$ des petites entreprises est un indicateur, contre $27,8 \%$ des entreprises de plus grande taille. On peut en conclure que les accords de coopération constituent des facteurs secondaires dans le processus d'internationalisation des PME locales. Comme indiqué dans cet article, les PME associent le concept d'internationalisation exclusivement à l'effort d'exportation.

Les données concernant la région environnante et la taille des entreprises qui ont conclu des accords de coopération permettent de faire ressortir les faits suivants :

- Les PME sont plus sensibles aux externalités locales.

- La coopération comme moyen d'internationalisation est un moyen pertinent seulement pour les grandes entreprises dans les systèmes locaux de production retenus.

3. Ces résultats ne discriminent pas les entreprises innovatrices de celles qui ne le sont pas, ni les entreprises exportatrices de celles qui ne le sont pas. 
- Les relations de coopération conclues à un niveau local semblent être formées entre des entreprises de même taille.

Les résultats obtenus révèlent des différences importantes entre les cas étudiés en Espagne et ceux des districts industriels en Italie qui ont fait l'objet d'une plus grande attention encore (Pyke, Becattini et Sengenberger, 1990). On peut noter que les marchés locaux de matériaux primaires et de composantes ont une importance significative dans les 23 régions étudiées, ce qui signifie que les entreprises réalisent des activités dans la transformation de ressources locales abondantes et développent des productions complémentaires.

À l'inverse, le marché local de la technologie n'est pas pertinent au regard de la taille (tableau 2). On peut considérer que les systèmes locaux étudiés ne peuvent pas fabriquer les équipements nécessaires à la production dans des conditions concurrentielles. Cela représente une faiblesse industrielle importante qui affecte l'autonomie dans l'innovation des procédés ainsi que dans la capacité de création de nouveaux produits pour le marché, c'est-à-dire la capacité de diversification du système de production local.

TABLEAU 2

Différents marchés d'approvisionnement des entreprises

\begin{tabular}{lccc}
\hline Marchés & $\begin{array}{c}\text { Matériaux } \\
\text { primaires }\end{array}$ & Composantes & Technologie \\
\hline Local (municipal/district) & 21,60 & 32,27 & 10,05 \\
Provincial & 17,32 & 19,79 & 8,39 \\
National & 51,48 & 40,40 & 43,16 \\
Reste du monde & 9,58 & 7,54 & 38,69 \\
Total des cas valides & & 205 & \\
\hline
\end{tabular}

Source : Costa, M.T. et al. (1993), op. cit.

Comme pour le marché d'information local, les effets de l'essaimage technologique local sont d'une importance considérable. Nous relevons ainsi que $82,8 \%$ des informations techniques acquises par l'entreprise proviennent du marché local. Il est intéressant de noter qu'un processus semblable à celui identifié par Von Hippel parmi les entreprises américaines est observé : l'information concernant les innovations et les changements dans le marché est diffusée à travers des échanges informels de connaissances entre les entrepreneurs.

Finalement, à partir d'un point de vue strictement territorial, les marchés de main-d'œuvre et de service locaux démontrent un niveau élevé d'autonomie, qui traduirait, tout au moins dans le premier cas, la cohésion entre le système social et le système de production de chaque territoire. 
La modalité dominante de production dans les systèmes de production locaux retenus est la fabrication en courtes séries de produits simples et complexes $(51,9 \%)$. En seconde place figure, loin derrière du premier modèle de production, la fabrication de prototypes et de produits personnalisés $(19,2 \%)$.

La structure industrielle des systèmes de production locaux de l'échantillon indique que $71,1 \%$ de la production n'engendrent pas d'économies d'échelle. Les seules régions où de longues séries de production de biens simples sont importantes sont celles de Béjar, Iscar, Murcia et Mataro. À l'autre extrémité, on a le cas de Vijo où $55 \%$ des entreprises fabriquent des prototypes et des produits spécialisés.

La production en courtes séries de produits complexes s'effectue selon des modèles organisationnels décentralisés dans $77,3 \%$ des cas étudiés, ce qui est sensiblement plus élevé que le taux observé pour l'ensemble des 324 entreprises. On note une intégration verticale plus poussée chez les entreprises ayant de longues séries de production. En outre, il existe une plus grande association de l'externalisation des fonctions à des modalités flexibles de production, laquelle peut être plus reliée à la capacité à obtenir de courtes séries qu'aux modalités de production de longues séries.

\section{GraPhiQue 1}

\section{Fréquence des modalités de production}

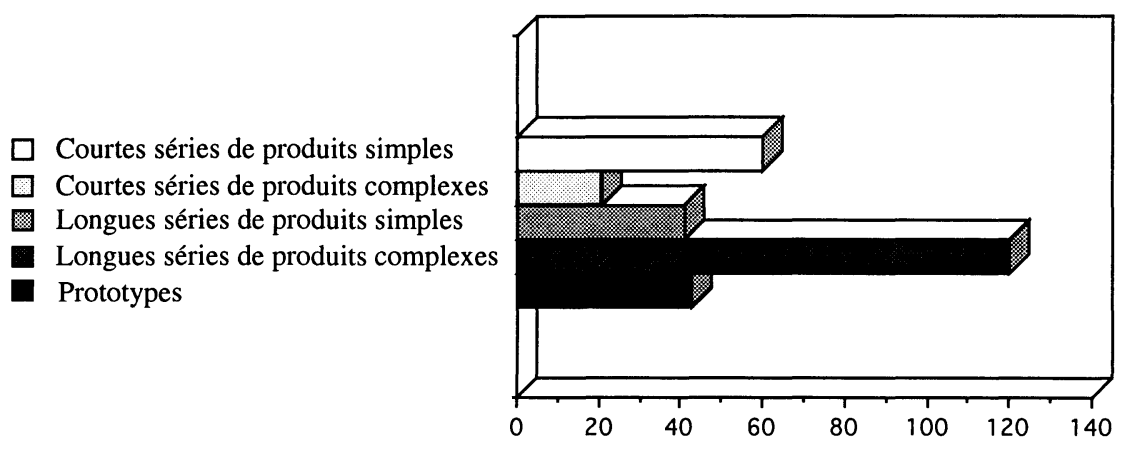

Source : Costa, M.T. et al. (1993), op. cit.

Toutefois, d'une façon particulièrement évidente, la flexibilité des courtes séries de produits complexes est moins associée à l'innovation organisationnelle que les modalités qui requièrent une plus grande intégration, comme dans le cas des longues séries de produits complexes. De cela, nous devons déduire que les externalités locales sont insuffisantes pour engendrer les niveaux 
d'innovation nécessaires pour soutenir la concurrence des marchés où les processus sont décentralisés et à courtes séries.

La décomposition de la fréquence de la collaboration entre les entreprises, avec des degrés différents d'externalisation, semble montrer, étant donné la taille des économies externes locales des systèmes de production étudiés, qu'un niveau d'externalisation supérieur à $50 \%$ donne lieu à une plus faible capacité de coopération.

Contrairement au domaine de la production où la coopération informelle prédomine, les accords de coopération entre les entreprises tendent à être formalisés, particulièrement dans les projets de R-D et dans la distribution.

Les formules les plus adoptées au sein de chaque fonction ou domaine sont les suivantes :

- R-D : accord temporaire entre entreprises $(38,7 \%)$ et constitution d'un groupe d'entreprises $(25,8 \%)$.

- Production: sous-traitance $(42,25 \%)$ et accord temporaire entre entreprises $(32,8 \%)$.

- Marketing : accord temporaire entre entreprises (39,3\%) et constitution d'un groupe d'entreprises $(32,1 \%)$.

- Services / assistance technique : constitution d'un groupe d'entreprises $(30,0 \%)$ et accords temporaires entre entreprises $(30,0 \%)$.

- Distribution : accords temporaires entre entreprises (61,5\%).

La forme la moins fréquente est la création de coentreprise qui représente $8,7 \%$ des accords de coopération étudiés.

Finalement, on peut noter que les séries courtes de produits simples et complexes présentent une faible capacité de coopération, qui pourraient être associées à la fois au nombre moins élevé de fonctions devant être coordonnées et aux limitations éventuelles du contexte environnant (capital humain, services locaux, associations entrepreneuriales) qui réduisent la possibilité de collaborer.

\subsection{Les facteurs externes locaux : les réseaux d'information}

Les réseaux d'information utilisés par les firmes qui ont entrepris une innovation de produit au cours des trois dernières années sont, par ordre d'importance, 1) les clients, 2) les foires commerciales et 3 ) les associations entrepreneuriales. Loin derrière se retrouvent les associations industrielles, les consultants externes et le personnel de l'entreprise elle-même. De la même manière, les réseaux d'information pour l'innovation de produit sont, par ordre d'importance, 1) le personnel technique à l'interne, 2) les foires commerciales et 3) les fournisseurs. 
Suivent plus loin les consultants externes, les associations industrielles et les entreprises dans le secteur et dans la région. Les aspects qui doivent être soulignés sont les suivants :

- l'importance des foires commerciales en tant que véhicule d'information dans tous les cas ;

- l'importance des clients dans l'innovation des produits;

- l'importance du personnel technique interne et des fournisseurs dans les innovations de procédés.

Les réseaux relevés ici semblent confirmer l'idée que les entreprises se réfèrent presque toujours aux innovations pour l'entité elle-même, plutôt qu'à des innovations originales dans le marché. Par conséquent, on peut expliquer pourquoi les foires commerciales, les clients et les fournisseurs tiennent une place fondamentale : c'est dans les foires commerciales que l'information sur l'évolution technique et le design des produits types de l'entreprise est acquise ; les clients expriment leurs désirs quant aux caractéristiques spécifiques des produits ; les fournisseurs offrent de plus en plus des machines et des matériels de pointe.

\subsubsection{La formation de la main-d'œuvre locale}

Les résultats obtenus montrent que les PME dans les systèmes choisis disposent d'un personnel plus qualifié que la moyenne en Espagne. Ce résultat est consistant avec les exigences de qualification d'une production flexible, qui est typique des modèles d'industrialisation locale.

La méthode de recrutement la plus utilisée par ces entreprises est le contact personnel. En outre, la confiance et l'appartenance à la même communauté semblent constituer des critères fondamentaux de recrutement. La flexibilité de la main-d'œuvre, comme l'utilisation de travailleurs à temps partiel, n'est pas fréquente. En retour, un degré élevé de flexibilité est observé au sein de chaque entreprise.

Le niveau plus élevé de qualification de la main-d'œuvre est aussi cohérent avec les difficultés plus grandes à recruter du personnel et l'activité de formation plus poussée. Il est particulièrement manifeste que les PME locales développent plus de programmes de formation que la moyenne des PME espagnoles. Ces activités de formation sont étroitement liées aux innovations des procédés et aux difficultés de recrutement. 
TABleau 3

Origine des employés au cours des trois dernières années

\begin{tabular}{lcc}
\hline Origine & $\begin{array}{c}\text { Directeurs } \\
\text { et techniciens }\end{array}$ & Autres employés \\
\hline Même municipalité & 63,0 & 75,1 \\
Reste du district & 26,8 & 23,4 \\
Reste de la communauté autonome & 6,6 & 0,6 \\
Reste de l'Espagne & 3,0 & 0,8 \\
Reste du monde & 0,6 & 0,1 \\
Total & 100,0 & 100,0 \\
\hline
\end{tabular}

Source : Costa, M.T. et al. (1993), op. cit.

\subsection{Les modèles d'internationalisation des systèmes industriels locaux}

L'internationalisation des systèmes industriels locaux se produit, dans la majorité des cas, à travers les mécanismes conventionnels d'exportation déployés par les entreprises, et fondamentalement, par celles qui produisent des biens de consommation dont l'avantage concurrentiel réside dans la marque ou le prix du produit final, ou les deux à la fois.

C'est le cas, entre autres, des entreprises Inca, Menorca, Ibi et Eda. C'est seulement dans un groupe restreint de régions, Bajo Deba, Castellon, Sabadell et Mansera, que l'avantage concurrentiel est déterminé par l'organisation des procédés complexes de production et se trouve associé à la qualité de la production finale.

Néanmoins, l'internationalisation, en termes de connaissance et d'adaptation des procédés de production et d'innovations de produits réalisées sur le marché international, peut se produire sans un effort important d'exportation. C'est la cas des activités fragmentées qui forment une partie du marché global en raison de la nature des composantes fabriquées. Cette modalité prévaut dans la région de Dos Hermanas Corridor, qui affiche peu d'efforts pour exporter $(0,33 \%)$. Les résultats de cette région, et à un moindre degré ceux de Mansera, montrent que les grandes entreprises peuvent affecter l'accélération du progrès technique et sur le développement des structures industrielles locales. Néanmoins, le maintien d'une relation de sous-traitance hiérarchique et exclusive réduit la capacité de négocier avec ses propres initiatives et tend à orienter l'accès au marché à moyen terme.

Les résultats obtenus de cette enquête confirment que, dans les systèmes locaux de production étudiés, l'innovation de produit, la conception, la commercialisation de sa propre marque et l'externalisation de la production des 


\section{TABLEAU 4}

\section{Différentes typologies de processus d'internationalisation}

Forte autonomie de
la grande entreprise
et des grands centres
de distribution

Hiérarchisation et plus grande dépendance des grandes entreprises ou des canaux de commercialisation

\section{Niveau des exportateurs}

Type 1a : Production flexible (Bajo Deba, Castellon).

Production flexible et autonomie élevée des entreprises dans les systèmes locaux de production. Diversification des marchés et produits de qualité élevée.

Type 2a : Production flexible et/ou concurrence globale (Sabadell, Mansera).

Diffère particulièrement des systèmes du type 3 a par les faibles niveaux de sous-traitance, un degré plus élevé d'autonomie et un effort d'exportation plus marqué.

Type 31 : Production flexible et/ou concurrence globale (Madrid Sud, Vigo, Iscar, Macael, Dos Hermanas Corridor).

Systèmes locaux de production à production flexible et/ou concurrence globale avec soustraitance limitée.
Type $1 \mathrm{~b}:$ Marque de production (Inca, Elda, Menorca, Ibi, Ubrique).

Marchés segmentés, diversifiés et très spécialisés. Politique de marque basée sur le design et la qualité. Autonomie de la grande entreprise et des canaux de commercialisation.

Type $2 b$ : Marque de production (Murcia, Orense, Alcoy).

Systèmes de production en phase de transition de $3 b$ à $1 b$.
Type $3 b$ : Production non différenciée (Mataro, Fuensalida, Urola, Béjar, Vallecas, Guijuelo).

Production peu différenciée et/ou avec forte sous-traitance. Hiérarchisation et dépendance à l'égard de grandes entreprises ou canaux de commercialisation. Ventes sur les marchés locaux régionaux.

Demande intermédiaire

Demande finale

Note méthodologique : La qualification de marque de commerce ou de production flexible vise à mettre en relief des facteurs d'avantage concurrentiel déterminants. Cela n'implique pas que la marque de production n'apparaît pas dans les systèmes locaux de production basés sur une production flexible ou que les systèmes locaux de production basés sur une production flexible ne peuvent pas implanter des politiques de nom de marque. Le passage à un niveau plus avancé s'accompagne d'une moindre différenciation et d'une moindre dépendance ainsi que d'une plus grande autonomie des entreprises dans les systèmes locaux de production.

Source: Costa, M.T. et al (1993), op. cit.

services sont des facteurs qui consolident la compétitivité des entreprises exportatrices. Concrètement, dans les systèmes locaux de production, ces caractéristiques sont liées aux entreprises qui exportent le plus par comparaison à celles qui exportent moins :

1) elles sont plus innovatrices dans le développement de nouveaux produits ;

2) elles accordent plus d'importance à la conception ; 
3) elles mettent l'accent sur la commercialisation de leurs propres marques, ce qui requiert l'utilisation d'un plus grand nombre de personnel affecté au marketing;

4) elles externalisent davantage les fonctions de production de services.

Dans le cas des entreprises qui exportent le plus, les accords de coopération permettent en particulier l'externalisation des fonctions marketing et de distribution. Ces accords tendent à être plus formels, contrairement aux accords de coopération informels ou aux relations de sous-traitance établies pour l'externalisation des phases de production.

On peut noter que la compétitivité sur le marché international des systèmes de production étudiés peut encore être expliquée en termes d'avantages comparatifs liés aux coûts et à la qualité des biens de consommation, classifiés par l'OCDE comme étant à faible demande. Autrement dit, la pénétration internationale des systèmes locaux de production prévaut seulement dans les marchés interindustries et, seulement d'une façon exceptionnelle, dans les relations internationales de nature intra-industrielle.

\section{Conclusion}

Cette étude empirique nous a permis de déterminer que les systèmes locaux de production présentent un niveau élevé de cohésion intra-industrielle et socioindustrielle. Les entreprises dans les systèmes locaux de production développent leurs processus de production et leurs innovations, particulièrement en relation avec les produits, dans des conditions d'efficacité et de compétitivité à un point tel qu'elles font partie des structures socio-industrielles locales. Dans ce sens, les économies externes (culturelles, sociales et industrielles) et la coopération entre les entreprises constituent des éléments de la cohésion avec le milieu environnant. Toutefois, les faiblesses relevées dans les mécanismes et les modèles d'internationalisation, dans les services aux entreprises, révèlent un champ d'action en matière de politique industrielle dont les résultats apparaissent décisifs pour l'avenir des systèmes locaux. En même temps, la mise en œuvre de telles actions ne devrait pas être considérée exclusivement en relation avec les systèmes de production locaux mais aussi en tant que programme visant la promotion de nouvelles régions, où les tendances de l'organisation industrielle devraient favoriser l'adaptation et la complémentarité entre le développement des systèmes locaux et les processus de globalisation. 


\section{Annexes}

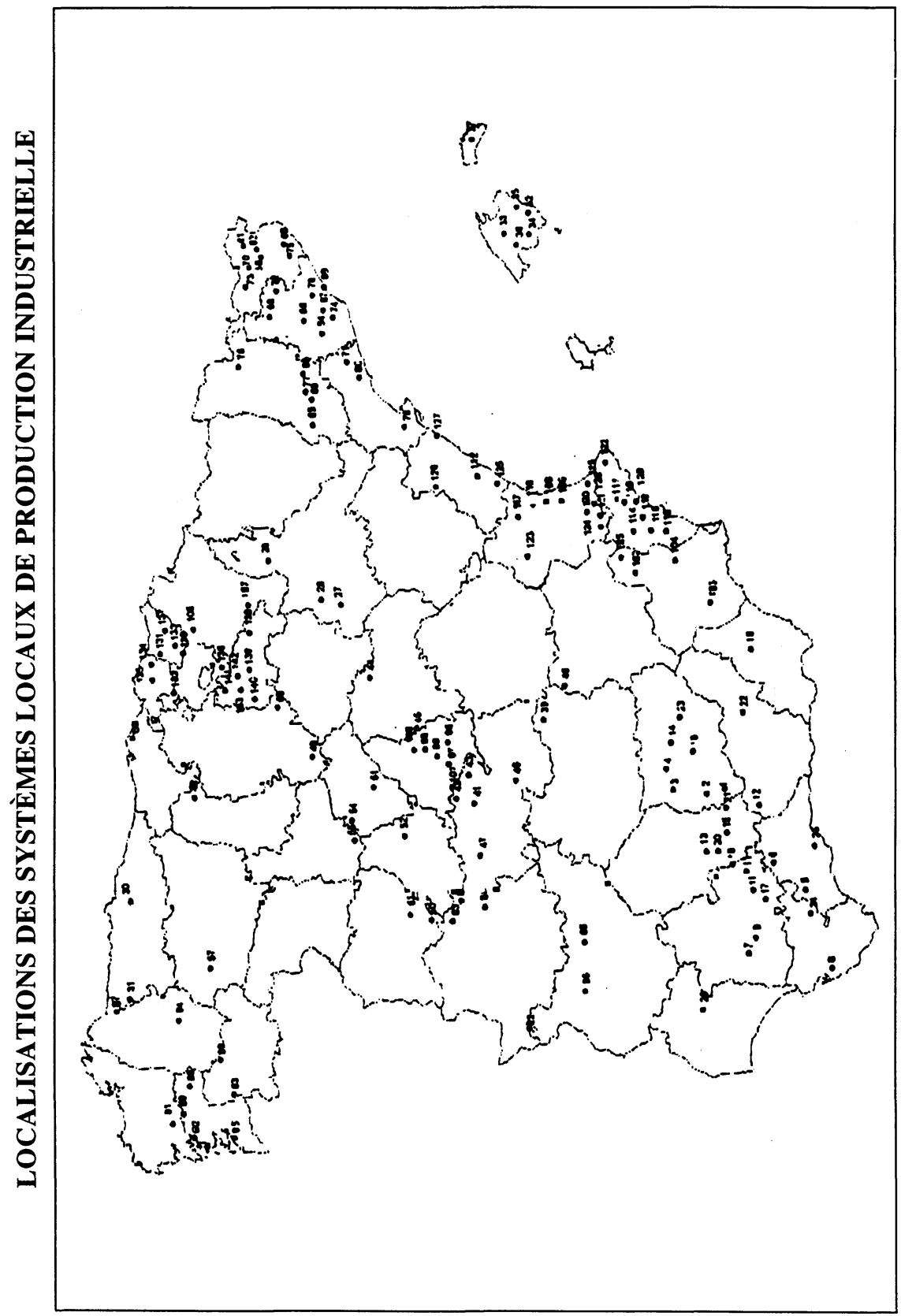




\section{LISTE DE LOCALISATION DES SYSTÈMES PRODUCTIFS LOCAUX INDUSTRIELS}

\begin{tabular}{|c|c|c|}
\hline ANDALOUSIE & CASTILLE-LÉON & 97. Área Sur \\
\hline 1. Alcalá la Real & 48. Aguilar del Campoó & (Fuenlabrada-Humanes) \\
\hline 2. Alcaudete & 49. Aranda del Duero & 98. Paracuellos \\
\hline 3. Andújar & 50. Béjar & 99. Vallecas \\
\hline 4. Bailén & 51. Cantimpalos & 100. Periféria regional noreste \\
\hline 5. Benaoján-Montejaque & 52. Cardeñosa & 101. Periféria regional sur \\
\hline 6. Campillos & 53. Guijuelo & MURCIE \\
\hline 7. Castilleja de la Cuesta & 54. Iscar & 102. Jumilla \\
\hline $\begin{array}{l}\text { 8. Chiclana de la Frontera } \\
\text { 9. Corredor de Dos Hermanas }\end{array}$ & $\begin{array}{l}\text { 55. Medina del Campo-Cuellar } \\
\text { 56. Quintanar de la Sierra }\end{array}$ & 103. Lorca \\
\hline 10. Estepa & 57. Ponferrada-El Barco & 104. Murcia (Molina de Segura) \\
\hline 11. Gilena & CATALOGNE & 10د. Yecla \\
\hline 12. Huétor-Tajar & 58. Banyoles & NAVARRE \\
\hline $\begin{array}{l}\text { 13. Rambla, La } \\
\text { 14. Linares-Carolina, La }\end{array}$ & 59. Berga & 106. Alsásua \\
\hline $\begin{array}{l}\text { 14. Linares-Carolina, La } \\
\text { 15. Lucena }\end{array}$ & 60. La Bisbal & 107. Ribera del Ebro \\
\hline 16. Mancha Real & 61. Figueres & PAYS VALENCIEN \\
\hline 17. Morón de la Frontera & 62. Girona & 108. Albal-Alfafar \\
\hline 18. Macael & 63. Guissona & 109. Alzira-Algemesi \\
\hline 19. Puebla de la Cazalla, La & 64. Igualada & 110. Agos \\
\hline 20. Puente Genil & $\begin{array}{l}\text { 65. Lleida } \\
66 \text { Manresa }\end{array}$ & 111. Alcoy \\
\hline $\begin{array}{l}\text { 21. Priego de Córdova } \\
\text { 22. Purullena }\end{array}$ & $\begin{array}{l}\text { 66. Manresa } \\
\text { 67. Martorell }\end{array}$ & 112. Castellón \\
\hline $\begin{array}{l}\text { 22. Purullena } \\
\text { 23. Úbeda }\end{array}$ & 68. Mataró & 113. Crevillente-Aspe \\
\hline $\begin{array}{l}\text { 23. Ubeda } \\
\text { 24. Ubrique }\end{array}$ & 69. Mollerussa & 114. Elda \\
\hline $\begin{array}{l}\text { 24. Ubrique } \\
\text { 25. Valle de Guadalhorce }\end{array}$ & 70. Olot & 115. Gandia \\
\hline $\begin{array}{l}\text { 25. Valle de Guadalhorce } \\
\text { 26. Valerde del Camino }\end{array}$ & 71. Penedès & 116. Ibi \\
\hline 20. Valerde del Camino & 72. Plana de Vic & $\begin{array}{l}\text { 17. Llirı-Beneguacil } \\
\text { 118. Manises }\end{array}$ \\
\hline ARAGON & 73. Ripoll & 119. Novelda \\
\hline 27. Calatayud & 74. Sabadell-Terrassa & 120. Otieria \\
\hline 28. Ejea de los & 75. La Selva & 121. Onteniente \\
\hline $\begin{array}{l}\text { Caballeros-Tauste } \\
\text { 29. Illueca-Brea }\end{array}$ & $\begin{array}{l}\text { 76. La Seu d'Urgell } \\
\text { 77. Tàrrega }\end{array}$ & $\begin{array}{l}\text { 122. Pedreguer-Gata de Gorgos } \\
\text { 123. Requena-Utiel }\end{array}$ \\
\hline ASTURIES & 78. Tortosa & 124. Vallada-Alberic \\
\hline 30. Norefia & 80. El Vendrell & $\begin{array}{l}\text { 125. Vall d'Uxó } \\
\text { 126. Villafranca-Portell }\end{array}$ \\
\hline BALÉARES & EXTRAMADURE & de Morella \\
\hline BALEAKES & 81. Navalmoral de la Mata & 127. Vinaroz-Benicarló \\
\hline $\begin{array}{l}\text { 32. Felanixt } \\
\text { 33. Inca }\end{array}$ & $\begin{array}{l}\text { 82. San Vicente de Alcántara } \\
\text { 83. Valle de Jerte }\end{array}$ & 129. Xixona \\
\hline 34. Llucmajor & 84. La Vera & PAYS BASQUE \\
\hline 35. Manacor & 85. Vega Alta & 130. Alto Deba \\
\hline 36. Marratxi & 86. Vega Baja & 131. Bajo Deba \\
\hline CANTARPF & GALICIE & 132. Cantabria Alavesa \\
\hline CANTABRE & 87. La Costa & 133. Goierri \\
\hline 38. Santoña-Laredo & 88. La Estrada & 134. Markina \\
\hline CASTILLE-LA MANCHE & 89. Lalin & 136. Rioja Alavesa \\
\hline 39. Alcázar de San Juan & $\begin{array}{l}\text { 90. Urense } \\
\text { 91. Padrón }\end{array}$ & 137. Urola \\
\hline $\begin{array}{l}\text { 40. Azuqueca de Henares } \\
\text { 41. Fuensalida }\end{array}$ & 92. Ria de Arosa & LA RIOJA \\
\hline 42. Méntrida & $\begin{array}{l}\text { 93. Rivadavia } \\
\text { 94. Sarna }\end{array}$ & 138. Arnedo \\
\hline 43. Sagra, La & 95. Vigo & 139. Baños del Rio Tobia \\
\hline 44. Sigüenza & & 140. Ezcaray \\
\hline 45. Socuéllamos-Almansa & MADRID & 141. Haro \\
\hline 46. Sonseca & 96. Arganda & 142. Nájera \\
\hline 47. Talavera de & & $\begin{array}{l}\text { 143. Santo Domingo } \\
\text { de la Calzada }\end{array}$ \\
\hline
\end{tabular}




\section{Bibliographie}

AOKI, M., B. GuSTAVSSON et O. WILliAMSON (1990), The firm as a nexus of treaties, Londres, Sage.

ARROW, Kenneth J. (1969), «The organization of economic activity : issues pertinent to the choice of market versus non market allocation », dans The Analysis and Evaluation of Public Expenditure : The PPB System, vol. 1, V.S. Joint Economic Committee, 91 st. Congress, 1 st., Session Washington, D.C., U.S. Government Printing Office, p. 59-73.

AXELrod, Robert (1984), La Evolución de la Cooperación, Madrid, Alianza Editorial.

BARNARD, Chester (1938), The Functions of the Executive, Cambridge, Harvard University Press.

BeCATtini, Giacomo (1975), Lo Sviluppo Economico della Toscana, Firenze, Guaraldi.

BECATtini, Giacomo (1979), «Dal settore industriale al distretto industriale. Alcune considerazioni sull'unitá di indagine dell economia industriale », Rivista di Economica e Politica Industriale, $\mathrm{n}^{0} 1$, p. 7-21.

Bellandi, Marco (1988), Economie di Scala e Costi di Transazione, Dipartamento di Scienza Economiche, Universitá degli studi di Firenze Gennaio, Mimeo.

BRUSCO, Sebastiano (1992), «Small firms and the provision of real services », dans F. Pyke et W. Sengenberger, Industrial Districts and Local Economic Regeneration, Genève, Institut international d'études sociales, p. 177-196.

CElADA, F. (1991), «Los sistemas productivos locales de carácter industrial en España », Madrid, Area y Systema.

COASE, R.H. (1937) «The nature of the firm », Economica, novembre, p. 386-405.

Costa, M. Teresa et al. (1993), Cooperación entre Empresas y Sistemas Productivos Locales en España. Una Aproximación, Madrid, MINER.

CostA, M. Teresa (1991), «La empresa española frente a la cooperación internacional », dans Juan Velarde, J.L. Garcia Delgado et Andrés Pedreño (éd.), Apertura e Internacionalización de la Economía Española, Madrid, Colleción Economistas, p. 397-416.

Dei Ottati, Gabi (1988), «El mercado comunitario del distrito industrial », Papers de Seminari, vol. 29-30, p. 180-218.

FERnÁNDEZ, E. et F. FERnÁNDEZ (1988), Manual de Dirección Estratégica de la Tecnología, Barcelone, Ed. Ariel.

GozzI, Antonio (1987), «Cambiamento della funzione di produzione estrategia di impresa », Economia e Politica Industriale, no 53, p. 109-129. 
KATZ, J. (1984), «Innovaciones technológicas internas y ventajas comparativas dinámicas : nuevas reflexiones sobre un programa de estudios casuísticos comparativos ", Información Commercial Española, $\mathrm{n}^{0} 605$.

Krugman, P. (1991), Geography and Trade, Cambridge, MIT Press.

Krugman, P. (1992), «Motivos y dificultades en la política industrial», dans C. Martin (éd.), Politica Industrial, Teoría y Práctica, Madrid, Economistas Libros.

LORENZONI, G. (1980), «Le strategi di imprese fondata su sinergie esterne », L'impresa, $\mathrm{n}^{0} 1$.

Marshall, Alfred (1980), Principles of Economics, 4e éd., Londres, MacMillan.

Pyke, F., G. Becattini et W. Sengenberger (1990), Industrial Districts and Interfirm Cooperation in Italy, Genève, Institut international d'études sociales.

Pyke, F. et W. Sengenberger (1992), Industrial Districts and Local Economic Regeneration, Genève, Institut international d'études sociales.

PORTER, Michael E. (1990), The Competitive Advantage of Nations, New York, Free Press.

SKINNER, W. (1978), Manufacturing in the Corporale Strategy, New York, John Wiley.

STIGLER, Georges J. (1951), «The division of labor is limited by the extent of the market », The Journal of Political Economy, juin, p. 185-193.

TANI, Piero (1987), «La descomponibilità del processo produttivo », dans Giacomo Becattini (éd.), Mercato e Forzi Locali : Il Distretto Industriale, Bologne, Mulino.

VON HIPPEL, E. (1987), «Cooperation between rivals : informal know-how trading », Research Policy, $\mathrm{n}^{0} 16$.

Williamson, Oliver (1975), Markets and Hierarchies : Analysis and Antitrust Implications, New York, Free Press.

Williamson, Oliver (1985), The Economic Institutions of Capitalism, New York, Free Press.

YounG, Allyn (1928), «Increasing returns and economic progress », The Economic Journal, décembre, vol. XXXVIII, no 152, p. 527-542.

ZEITLIN, Jonathan (1992), «Industrial districts and local economic regeneration: overview and comment », dans F. Pyke et W. Sengenberger, op. cit. 